\title{
METRIC ENTROPY OF CERTAIN CLASSES OF LIPSCHITZ FUNCTIONS ${ }^{1}$
}

\author{
A. C. VOSBURG
}

1. Introduction. In this paper we discuss the metric entropy (in the uniform metric) of certain classes $\operatorname{Lip}(\alpha / A)$ of Lipschitz functions which will be defined in $\$ 2$.

The notion of metric entropy (or $\epsilon$-entropy) of a totally bounded subset $A$ of a metric space was introduced by Kolmogorov [1] to characterize the massiveness of $A$. Among the most striking applications of this notion are the results of Kolmogorov [3] and Vitushkin [7]; for expositions of the subject of metric entropy see Lorentz [4], $[5]$.

We collect some basic definitions and facts. $A$ will always denote a nonempty subset of a metric space $X$, and $\epsilon$ a positive number not exceeding unity. We use the notation $f(\epsilon) \sim g(\epsilon)$ to mean $\lim (f(\epsilon) / g(\epsilon))$ $=1$ as $\epsilon \rightarrow 0+$ and $f(\epsilon) \ll g(\epsilon)$ to mean $f(\epsilon)=O(g(\epsilon))$ as $\epsilon \rightarrow 0+; f(\epsilon)$ $\approx g(\epsilon)$ means that both $f(\epsilon) \ll g(\epsilon)$ and $g(\epsilon) \ll f(\epsilon)$. All logarithms will have base 2 .

Definition 1. A class $C$ of subsets of $X$ is called an $\epsilon$-cover of $A$ if each set in $C$ has diameter not exceeding $2 \epsilon$ and $A \subset \cup\{C: C \in C\}$.

Definition 2. A subset $D$ of $X$ is called $\epsilon$-distinguishable if the distance between each pair of points of $D$ exceeds $\epsilon$.

Definition 3. A subset $N$ of $X$ is called an $\epsilon$-net for $A$ if each point of $A$ is within distance $\epsilon$ of some point of $N$.

For totally bounded sets $A$ (i.e., sets having a finite $\epsilon$-cover for each $\epsilon>0$ ) we make the following definitions which are due to Kolmogorov [3].

Definition 4. $N_{\epsilon}(A)$ denotes the minimal number of sets in any $\epsilon$-cover of $A$.

Definition 5. $M_{\epsilon}(A)$ denotes the maximal number of points in any $\epsilon$-distinguishable subset of $A$.

Definition 6. $H_{\epsilon}(A)=\log N_{\epsilon}(A)$ is called the e-entropy of $A$.

Definition 7. $C_{\epsilon}(A)=\log M_{\epsilon}(A)$ is called the $\epsilon$-capacity of $A$.

The following basic theorem of Kolmogorov [3, $\$ 1]$ will be used.

Received by the editors December 12, 1965.

1 This paper is related to part of the author's doctoral dissertation which was directed by Professor George G. Lorentz and supported by the United States Air Force through the Air Force Office of Scientific Research, under Contract No. AF 49(638)1401. 
Theorem. Let $A$ be a totally bounded subset of a metric space $X$, $\epsilon>0$. Then

$$
M_{2 \epsilon}(A) \leqq N_{\epsilon}(A) \leqq M_{\epsilon}(A)
$$

and hence also

$$
C_{2 \epsilon}(A) \leqq H_{\epsilon}(A) \leqq C_{\epsilon}(A) .
$$

It should also be noted that for $A \subset(-\infty,+\infty)$

$$
N_{\epsilon}(A) \leqq T N_{T \epsilon}(A)
$$

holds for each integer $T \geqq 1$ (each set which covers $A$ and has diameter $\leqq T \epsilon$ can be replaced by $T$ sets of diameter $\leqq \epsilon)$.

2. The entropy of $\operatorname{Lip}(\alpha / A)$. In the following $A$ will always denote a compact subset of $[0,1]$. All function spaces considered will have the uniform metric.

Definition 8. Let $\alpha>0$ and let $A$ be a compact subset of $[0,1]$. By $\operatorname{Lip}(\alpha / A)$ is meant the (compact) set of all real valued functions defined on $A$ for which $\left|f(x)-f\left(x^{\prime}\right)\right| \leqq\left|x-x^{\prime}\right|^{\alpha}$ for all $x, x^{\prime}$ in $A$ and $\max _{x \in A}|f(x)| \leqq 1$.

It will be convenient to consider $\operatorname{Lip}(\alpha / A)$ as a subspace of $M(\alpha / A)$, the space of all functions on $A$ for which $\left|f(x)-f\left(x^{\prime}\right)\right| \leqq 5\left|x-x^{\prime}\right|^{\alpha}$ for all $x, x^{\prime}$ in $A$ and $\max _{x \in A}|f(x)| \leqq 2$. We investigate how $H_{\epsilon}(\operatorname{Lip}(\alpha / A))$ depends upon $A$. For the case $A=[0,1]$, Kolmogorov and Tihomirov $[3]$ have shown

$$
H_{\epsilon}(\operatorname{Lip}(\alpha / A)) \approx \epsilon^{-1 / \alpha} .
$$

Also, it is easy to establish $[3, \S 9(235)$, p. 354] that for any nonempty totally bounded set $B$ in a metric space (letting $\delta=\epsilon^{1 / \alpha}$ )

$$
H_{\epsilon}(\operatorname{Lip}(\alpha / B)) \gg N_{\delta}(B)+\log \epsilon^{-1} .
$$

Related estimates which will not be needed here can be found in [6] and $[8, \S 17]$.

In the following we obtain an upper estimate for $H_{\epsilon}(\operatorname{Lip}(\alpha / A))$ which leads to a simple characterization of compact subsets $A$ of $[0,1]$ for which (4) holds.

TheOREM 1. Let $A$ be a compact subset of $[0,1]$, and assume 0 $<\alpha \leqq 1$. Then, letting $\delta=\epsilon^{1 / \alpha}$, we have

$$
H_{\epsilon}(\operatorname{Lip}(\alpha / A)) \ll N_{\delta}(A) \cdot \log \left(2 \epsilon^{-1}\left(N_{\delta}(A)\right)^{-\alpha}\right)+\log \epsilon^{-1} .
$$

Proof. Let $\epsilon(0<\epsilon<1)$ be given and let $A_{0}=\left\{x_{1}, x_{2}, \cdots, x_{M}\right\}$ be (from left to right) a maximal set of $\delta / 2$-distinguishable points of 
$A$ ( $M$ abbreviates $M_{\delta / 2}(A)$, and we can assume $M \geqq 2$ ), and for each $i=1,2, \cdots, M$, let $I_{i}$ be the closed interval centered at $x_{i}$ having length $\delta$. The class $\left\{I_{i}: i=1,2, \cdots, M\right\}$ covers $A$, since otherwise $A_{0}$ would not be maximal.

Let $F_{0}$ denote the family of functions obtained by restricting the functions of $\operatorname{Lip}(\alpha / A)$ to $A_{0}$. Further, for each $h \in F_{0}$, let $f_{h}$ be a function defined on $A_{0}$ such that $f_{h}\left(x_{i}\right)$ is an integral multiple of $\epsilon$ and $\left|f_{h}\left(x_{i}\right)-h\left(x_{i}\right)\right| \leqq \epsilon$ for each $i=1,2, \cdots, M$. Let $F^{*}$ denote the set of functions so obtained. It is easy to see that $F^{*} \subset M\left(\alpha / A_{0}\right)$. In fact, letting $f_{h}$ be an arbitrary function of $F^{*}$ we have, for any $x_{i}, x_{j}$ in $A_{0},\left|f_{h}(x)\right| \leqq\left|f_{h}(x)\right|+\epsilon \leqq 1+1$ and $\left|f_{h}\left(x_{i}\right)-f_{h}\left(x_{j}\right)\right| \leqq\left|h\left(x_{i}\right)-h\left(x_{j}\right)\right|$ $+2 \epsilon=\left|h\left(x_{i}\right)-h\left(x_{j}\right)\right|+2 \delta^{\alpha} \leqq\left|x_{i}-x_{j}\right|^{\alpha}+2 \delta^{\alpha} \leqq\left|x_{i}-x_{j}\right|^{\alpha}+2^{\alpha+1}\left|x_{i}-x_{j}\right|^{\alpha}$ $\leqq 5\left|x_{i}-x_{j}\right|^{\alpha}$; hence $F^{*} \subset M\left(\alpha / A_{0}\right)$. Further let $F$ denote a subset of $M(\alpha / A)$ obtained by extending each function of $F^{*}$ to $A$. It is easy to see that such an $F$ exists (since $A_{0}$ is finite, each function of $M\left(\alpha / A_{0}\right)$ can be extended linearly to be a function of $\left.M(\alpha / A)\right)$.

Now let $n(F)$ denote the number of elements of $F$. Then $n(F)$ $\geqq N_{14 \epsilon}(\operatorname{Lip}(\alpha / A))$, which can be seen as follows. Let $g \in \operatorname{Lip}(\alpha / A)$ and let $f$ be a function of $F$ which at each $x_{i}(i=1,2, \cdots, M)$ has values no farther than $\epsilon$ from those of $g$. Let $a \in A$, so $a \in I_{i}$ for some $i=1,2, \cdots, M$. Then we have $|f(a)-g(a)| \leqq\left|f(a)-f\left(x_{i}\right)\right|+\mid f\left(x_{i}\right)$ $-g\left(x_{i}\right)|+| g\left(x_{i}\right)-g(a) \mid \leqq 5\left(\epsilon^{1 / \alpha}\right)^{\alpha}+\epsilon+\left(\epsilon^{1 / \alpha}\right)^{\alpha}=7 \epsilon$. Hence $\|f-g\|$ $=\max _{a \in A}|f(a)-g(a)| \leqq 7 \epsilon$, showing that $F$ is a $7 \epsilon$-net for $\operatorname{Lip}(\alpha / A)$. Since the family of spheres of diameter $14 \epsilon$ centered at the points of $F$ covers $\operatorname{Lip}(\alpha / A), N_{14 \epsilon}(\operatorname{Lip}(\alpha / A))$ cannot exceed $n(F)$.

It remains to estimate $n(F)$ from above. To do this let $f \in F$ and note that there are no more than $[4 / \epsilon]+1$ possible values of $f\left(x_{1}\right)$; for each of these there are no more than $\left[2\left(x_{2}-x_{1}\right)^{\alpha} \epsilon^{-1}\right]+1^{2}$ possible values of $f\left(x_{2}\right)$, and, in general, for each $k=1,2, \cdots, M-1$, there are no more than $\left[2\left(x_{k+1}-x_{k}\right)^{\alpha} \epsilon^{-1}\right]+1$ possible values of $f\left(x_{k+1}\right)$. Furthermore, for each $k=1,2, \cdots, M-1$, we have $\left[2\left(x_{k+1}-x_{k}\right)^{\alpha} \epsilon^{-1}\right]$ $+1 \leqq 4\left(x_{k+1}-x_{k}\right)^{\alpha} \epsilon^{-1}$ because $2 x+1 \leqq 4 x$ if $x \geqq 1 / 2$ (in our case $x=\left(x_{k+1}-x_{k}\right)^{\alpha} \epsilon^{-1} \geqq 1 / 2$ because $A_{0}$ is $(1 / 2) \epsilon^{1 / \alpha}$-distinguishable and hence, since $0<\alpha \leqq 1,(\epsilon / 2)^{1 / \alpha}$-distinguishable). Thus $n(F)$ does not exceed

$$
([4 / \epsilon]+1) 4^{M-1} \epsilon^{-(M-1)} \prod_{k=1}^{M-1}\left(x_{k+1}-x_{k}\right)^{\alpha} .
$$

And since any product $y_{1} \cdot y_{2} \cdot \ldots \cdot y_{M-1}$ subject to the conditions $y_{i}>0(i=1,2, \cdots, M-1)$ and $\sum_{i=1}^{M-1} y_{i}=$ constant is maximized by

$2[x]$ denotes the largest integer not exceeding $x$. 
taking $y_{i}=y_{2}=\cdots=y_{M-1},(7)$, and hence also $N_{14 \epsilon}(\operatorname{Lip}(\alpha / A))$, does not exceed $5 \epsilon^{-1}\left(4 \epsilon^{-1}(M-1)^{-\alpha}\right)^{M} \leqq 5 \epsilon^{-1}\left(8 \epsilon^{-1} M^{-\alpha}\right)^{M}$.

Thus, letting $N$ abbreviate $N_{\delta / 2}(A)$ and using (1) and (3),

$$
\begin{aligned}
H_{14 \epsilon}(\operatorname{Lip}(\alpha / A)) & \leqq \log 5 \epsilon^{-1}+M \log \left(8 \epsilon^{-1} M^{-\alpha}\right) \\
& \leqq \log 5 \epsilon^{-1}+N_{\delta / 4}(A) \log \left(8 \epsilon^{-1} N^{-\alpha}\right) \\
& \ll N_{\delta}(A) \cdot \log \left(2 \epsilon^{-1}\left(N_{\delta}(A)\right)^{-\alpha}\right)+\log \epsilon^{-1}
\end{aligned}
$$

follows immediately, which concludes the proof of the theorem.

The maximum possible value of $N_{\epsilon}(A)$ for sets $A \subset[0,1]$ occurs when $A=[0,1]$, in which case $\epsilon^{-1} \leqq N_{\epsilon}(A) \leqq \epsilon^{-1}+1$; the relation $N_{\epsilon}(A)=o\left(\epsilon^{-1}\right)$ means that $A$ is in some sense rarified in $[0,1]$. Likewise, the maximum possible value of $H_{\epsilon}(\operatorname{Lip}(\alpha / A))$ for $A \subset[0,1]$ is $H_{\epsilon}\left(\operatorname{Lip}(\alpha /[0,1]) \approx \epsilon^{-1 / \alpha}\right.$. The following theorem shows that for sets $A$ which are rarified in $[0,1], H_{\epsilon}(\operatorname{Lip}(\alpha / A))$ cannot reach its maximum; it also characterizes when this maximum is achieved. First we need the following lemma.

Lemma 1. Let $A$ be a compact subset of $[0,1]$.

(i) If meas $A>0$ then $N_{\epsilon}(A) \approx \epsilon^{-1}$

(ii) If meas $A=0$ then $N_{\epsilon}(A)=o\left(\epsilon^{-1}\right)$.

Proof. For any interval $I$ of length $l$ we have $l /(2 \epsilon) \leqq N_{\epsilon}(A)$ $<l /(2 \epsilon)+1$. From the definitions of $N_{\epsilon}(A)$ and Lebesgue measure

$$
\operatorname{meas}(A) /(2 \epsilon) \leqq N_{\epsilon}(A) \leqq N_{\epsilon}[0,1]<1 /(2 \epsilon)+1 .
$$

This proves (i).

To prove (ii), suppose meas $A=0$ and let $\delta>0$ be arbitrary. Then there exist finitely many intervals $I_{i}$ of lengths $l_{i}, i=1,2, \cdots, k$, which cover $A$ and for which $\sum_{i=1}^{k} l_{i} \leqq \delta$. Letting $A_{i}=A \cap I_{i}$, we have

$$
N_{\epsilon}(A) \leqq \sum_{i=1}^{k} N_{\epsilon}\left(A_{i}\right) \leqq \sum_{i=1}^{k}\left(l_{i} / 2 \epsilon+1\right) \leqq \delta / 2 \epsilon+k<\delta / \epsilon
$$

for all sufficiently small $\epsilon>0$, which concludes the proof of the lemma.

Theorem 2. Let $A$ be a compact subset of $[0,1]$. If meas $(A)>0$, then

$$
H_{\epsilon}(\operatorname{Lip}(\alpha / A)) \approx \epsilon^{-1 / \alpha}
$$

if meas $(A)=0$, then

$$
H_{\epsilon}(\operatorname{Lip}(\alpha / A))=o\left(\epsilon^{-1 / \alpha}\right) .
$$

Proof. Let $\delta=\epsilon^{1 / \alpha}$. If meas $(A)>0$, then Lemma 1 yields $N_{\delta}(A)$ $\approx 1 / \delta$. Using (5) and (6) this yields $H_{\epsilon}(\operatorname{Lip}(\alpha / A)) \approx N_{\delta}(A)+\log \epsilon^{-1}$ $\approx \epsilon^{-1 / \alpha}$. 
If meas $(A)=0$, then for $T_{\mathbf{c}}=\delta N_{\delta}(A)$ we have, by Lemma 1 , $T_{\epsilon}=o(1)$.

Therefore, by (6),

$$
\begin{aligned}
H_{\epsilon}(\operatorname{Lip}(\alpha / A)) & \ll \delta^{-1} T_{\epsilon} \log \left(2\left(T_{\epsilon}\right)^{-\alpha}\right)+\log \epsilon^{-1}=o\left(\delta^{-1}\right)+\log \epsilon^{-1} \\
& =o\left(\epsilon^{-1 / \alpha}\right),
\end{aligned}
$$

which completes the proof of the theorem.

\section{REFERENCES}

1. A. N. Kolmogorov, Asymptotic characteristics of some completely bounded metric spaces, Dokl. Akad. Nauk SSSR 108 (1956), 585-589.

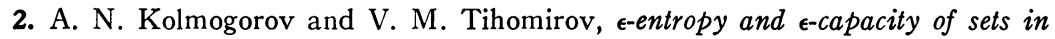
function spaces, Uspehi Mat. Nauk 14 (1959), 3-86.

3. - $-\epsilon$-entropy and $\epsilon$-capacity of sets in functional spaces, Amer. Math. Soc. Transl. (2) 17 (1961), 277-364.

4. G. G. Lorentz, Approximation of functions, Holt, Rinehart and Winston (to appear).

5. - Metric entropy, widths, and superpositions of functions, Amer. Math. Monthly 69 (1962), 469-485.

6. A. F. Timan, On the order of magnitude of $\epsilon$-entropy of spaces of real continuous functions defined on connected compact sets, Uspehi Mat. Nauk (115) 19 (1964), 173177.

7. A. G. Vitushkin, Estimation of the complexity of tabulation problems, Gosizdat, Moscow, 1959.

8. - Theory of the transmission and processing of information, Pergamon Press, New York, 1961.

SyRACUSE UNIVERSITY AND

The General Electric Company 